\title{
The Use of Convolutional Neural Network LeNet for Pollen Grains Classification
}

\author{
A. Korobeynikov ${ }^{1}$, Yu. Kamalova ${ }^{2}$, M. Palabugin ${ }^{3}$, I. Basov ${ }^{4}$ \\ ${ }^{1}$ Dept. of Computer Software, \\ ${ }^{2}$ Dept. of Measuring, Control, Diagnostic Instruments and Techniques, \\ ${ }^{3}$ Dept. Physics and Optical Engineering, \\ ${ }^{4}$ Dept. Mathematical Support for Information Systems, \\ Kalashnikov Izhevsk State Technical University, \\ Izhevsk, Russian Federation \\ E-mail: ${ }^{1}$ kav33@inbox.ru, ${ }^{2}$ julyprudent@mail.ru, ${ }^{3}$ mien@istu.ru, ${ }^{4}$ mien@istu.ru
}

Received: June 26, 2018

\begin{abstract}
The convolutional neural network from the LenetMnistExample of the DeepLearning4j framework is described and applied for pollen grains classification. The selected basic topology of the LeNet neural network was not changed; the loading of images was modified, and the number of classes of the classification task (outputNum) as well as the subsample size of examples (batchSize) were changed. Training (1520 photos) and test (380 photos) samples of four classes of pollen grains were formed. The quality metrics values calculated according to the results of the test sample classification are: 1) Accuracy $=0.9289 ; 2)$ Precision $=0.9306 ; 3)$ Recall $=0.9266 ; 4)$ F1 Score $=0.9282$.
\end{abstract}

Keywords: computer pollen analysis, pollen of plants, image recognition, convolutional neural network, pollen recognition.

\section{INTRODUCTION}

In modern society, the trend towards automation and computerization of various technological processes is becoming increasingly prevalent. The number of areas using computer analysis for the recognition of various objects is very significant and continues to increase. And one of the promising areas using this technology is pollen analysis. Nowadays, there are a number of studies based on the recognition of pollen grains, determining their characteristics.

In the article [1] authors researched an automation of the process for the classification of pollen grains. An updated set of three schemes of pollen extractors and four algorithms for machine learning were implemented. This is CST, BOW, CST + BOW. With the usage of the $\mathrm{BOW}+\mathrm{CST}$ method and the C-SVC classifier, the percentage of correct classifications reached $94 \%$. This was the best result in this research. BOW and CST individually had low accuracy.

In the work [2] there is described a semiautomatic system for pollen grains analysis with the usage of computer vision for increasing the number of processed data. Computer vision

(C) Korobeynikov A., Kamalova Yu., Palabugin M., Basov I., 2018 
algorithms was used for automation. High performance was achieved with minimal parameter settings.

The authors of the paper [3] developed a method based on the detection of small shifts at the absolute direct current level of the cantilever deflection signal that occurs when the body is tied to the tip during atomic force microscopy. The addition of recognition images gives the microscope the ability to identify certain types of molecules at the level of one molecule without compromising their topographic characteristics.

In the article [4] there are results for creating an automatic system that produces microscopic analysis of biological particles. As a basis, the authors took an analytical approach. This system has yielded good results in the collection of pollen data. The accuracy of detecting pollen grains using this method is $90 \%$.

In the paper [5] authors carried out research in the field of automatic recognition of pollen grains. In their work they used the OpenCV recognition library and the method of point marks on the object. Accordingly, the neural network correctly recognized about $72 \%$ of the pollen grains.

Authors of the work [6] carried out work on the development of automated recognition of pollen. The AMIT Leitz Ergolux AMC trinocular microscope was used, which was equipped with a stepped step X-Y-Z with computer-controlled Quick Step. To solve the problem associated with an intensive computational process of image processing, this work used a hierarchical approach. In this paper, for the first time, a system was provided that has the potential to produce slides made with a microscope that contain pollen grains.

In the paper [7] authors conducted an exhaustive analysis of the conformity of the texture of the pollen grains and its taxon. They used the method of textures - an effective method for identifying pollen. The results reflect the excellent characteristics of the textures descriptors LGF and DTM, as well as the usefulness of the combined approach in the proposed problem of pollen classification. A significant classification accuracy of more than $80 \%$ has been achieved.

Author of the paper [8] developed software for computer vision that can help researchers in the classification and analysis of pollen. Using the FLLT method, which makes up the implicit contours of the object, after which the segmentation procedure is performed. And then it is possible to classify pollen grains. In his view, a two-stage method is required for computerclassical classification of images. Fourier descriptors using centroid distance have been defined as an ideal way of determining the similarity of a figure.

In the work [9] authors used a method for classifying pollen grains, which involves determining the size and density of sculptural elements grain, also paying attention to the complexity of the relief of the surface of the pollen. The results of the studies support the theory that a combination of high resolution microscopy and analysis of computational images can generate a classification at subtle taxonomic levels that are not available to specialists. In the paper, they also carried out the classification of textures using histograms of local quantum spots of images, which gave an accuracy of more than $85 \%$, but did not provide high interpretability.

Authors in paper [10] carried out work on the automation of differential blood counting (DBC). An effective automatic system for differential blood analysis was developed. It does not require user settings and any intervention in the human process. The segmentation scheme used in the system can be easily adapted for any given data set with a known magnification. However, for this method to be clinically useful, it is necessary to improve the process associated with the processing of clustered data cells. The database of blood bodies was obtained from the database of stained May-Grunwald Giemsa stains (IGY). The classification accuracy was $80 \%$.

Authors in the article [11] carried out research to automate the processing of data associated with pollen. They describe a method for classifying pollen that uses different grain attributes in the multi-layered decomposition of areas in the pollen image. Here researchers use a hierarchical classification. This method resulted in one of the highest classification rates. At the first stage, the 
method first classifies pollen types into two broad groups, depending on the appearance of the texture. In the second stage, the method classifies the pollen using the decomposition method, which creates several layers for each sample. The accuracy of the method is $80 \%$.

As we see attempts for automation of the process of classification of pollen grains on microphotographs were made. Some of them has a high accuracy of recognition. However, the problem wasn't completely solved, i.e. until now there is no method for determining the botanical and geographical origin of honey and other bee products, based on a fully computer analysis of pollen grains.

Determining the grain's belonging to the plant species by a highly qualified operator is an expensive procedure, but it is still the most accurate and effective one.

The relevance of the use of computer analysis in the control of honey can be explained, firstly, by the possibility of determining the fact of falsification of honey by identifying the correspondence between the name of honey and the belonging of pollen grains to a particular plant. It can find its application in the provision of services for analyzing the quality of honey, its belonging to a particular monoflorous sort of honey that will allow issuing certificates confirming the quality of honey.

Computer pollen analysis is relevant in many areas of science and production, such as control of botanical and geographical honey origin, pharmaceutical industry, ecology, palinology, etc.

Palynological data are used in a wide range of areas, but the problems of classification and counting of pollen grains require high qualification and labor intensity. The development of an automated pollen identification and classification system will bring great benefits. Previous attempts at computer classification have used approaches that by nature were difficult to develop into fully automated systems able to operate independently to a considerable extent.

In this work, to solve the pollen grains classification problem, the convolutional neural networks were chosen, as they have been successfully used for image processing in recent years [12-14].

Artificial neural network of Fukushima (Cognitron, 1975) [15] was the first convolutional artificial neural network, which borrowed the ideas embodied by nature in the visual cortex for informatics. The convolutional network in its modern form appeared in the works of LeCun (LeNet, 1989) [15].

\section{THE METHOD OF THE EXPERIMENT}

As a base, the artificial neural network topology was chosen from the example of the LenetMnistExample of DeepLearning4j framework ${ }^{1}$. In this example, the classification problem of handwritten digits images with size of $28 \times 28$ from the MNIST database ${ }^{2}$ training sample is solved.

During experiments, the selected basic topology of the LeNet neural network was not changed; the loading of images was modified, and the number of classes for the classification task (outputNum) and the subsample size of examples (batchSize) were changed.

Typical image processing using convolutional neural network LeNet is shown in Fig. 1 [16], where the feature maps (matrixes) obtained at the outputs of sequential layers of the neural network are shown schematically.

The following parameters of the LeNet neural network layers were used in the experiments:

\footnotetext{
${ }^{1} \mathrm{http}: / /$ DeepLearning4j.org

${ }^{2} \mathrm{http}$ ://yann.lecun.com/exdb/mnist/
} 
0 . Input image: size of $28 \times 28,1 \mathrm{RGB}$ colors layer. The size of the input multidimensional array is $28 \times 28 \times 1$.

1. Convolutional layer (Convolutions): the convolution kernel size of $3 \times 3$, the number of different convolutions (feature filters) 20, linear neuron activation function (Identity). At the layer output a multidimensional array of size $28 \times 28 \times 20$ is derived.

2. Subsampling layer (Subsampling): the kernel size of $2 \times 2$, maximum function (Max). At the layer output a multidimensional array of size $14 \times 14 \times 20$ is derived.

3. Convolutional layer (Convolutions): the convolution kernel size of $5 \times 5$, the number of different convolutions (feature filters) 50 , linear neuron activation function (Identity). At the layer output a multidimensional array of size $14 \times 14 \times 50$ is derived.

4. Subsampling layer (Subsampling): the kernel size of $2 \times 2$, maximum function (Max). At the layer output a multidimensional array of size $7 \times 7 \times 50$ is derived.

5. Dense layer (Fully connected): number of neurons 500, ReLU (Rectified Linear Unit) neuron activation function. At the layer output an array of size 500 is derived.

6. Output layer (Output): number of neurons (classes) 4, SoftMax neuron activation function. At the layer output a array of size 4 is derived.

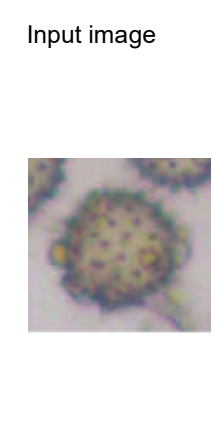

$28 \times 28 \times 1$

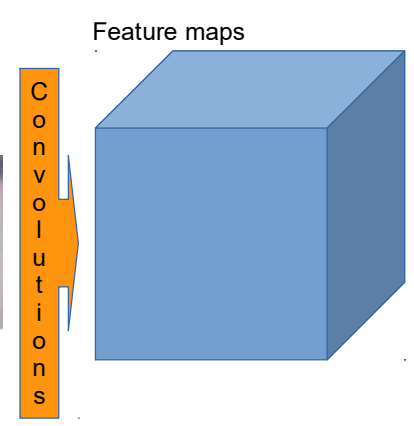

$28 \times 28 \times 20$

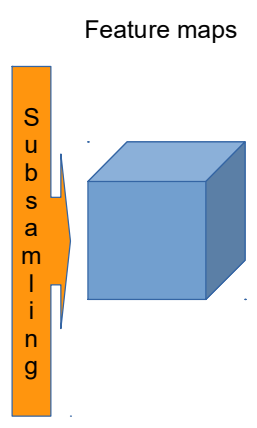

$14 \times 14 \times 20$

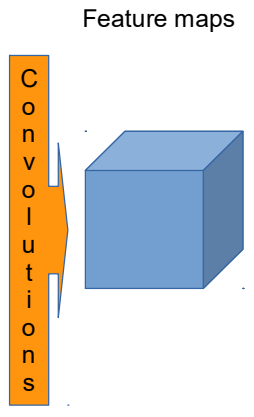

$14 \times 14 \times 50$

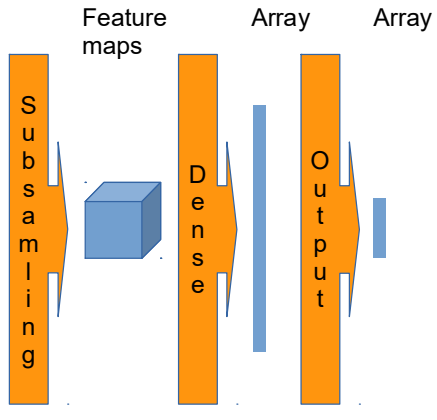

$7 \times 7 \times 50$
500
4

Figure 1. Image processing by convolutional neural network

\section{EXPERIMENTS}

Block diagram used for experiments is shown in Fig. 2. The following parameters were used during the training of convolutional neural network: 1) number of output neurons: 4; 2) the subsample size of examples (batchSize): 128; 3) number of training epochs (epochNum): 1000; 4) the number of iterations at each epoch: 1; 5) L2 regularization weight: $0.0005 ; 6$ ) learning rate: 0.01 . For fitting the neural network was used the optimization algorithm "stochastic gradient descent" with updater "Nesterovs".

During the experiments, a pollen grains sample containing 4 classes of grains was used: 0) Centaurea scabiosa; 1) Helianthus; 2) Pisum; 3) Fagopyrum. Examples of images from each class are shown in Fig. 3. All images were split into training and test samples containing about $80 \%$ and $20 \%$ of images from each class respectively. Table 1 shows the number of images from each class in the samples.

The results of the test sample classification obtained during the experiments are presented in Table 2. Let's consider the data in table 2 for class 0: a total of 88 samples from this class are in the test sample, of these, the neural network related 79 samples to class 0,1 sample to class 1 and 8 samples to class 2 . The quality metrics values calculated according to the results of the test sample classification are: 1 ) accuracy $=0.9289 ; 2$ ) precision $=0.9306 ; 3$ ) recall $=$ $=0.9266$; 4) F1 score $=0.9282$. 
"Instrumentation Engineering, Electronics and Telecommunications - 2018"

Proceedings of the IV International Forum (Izhevsk, Russia, December, 12-14, 2018)

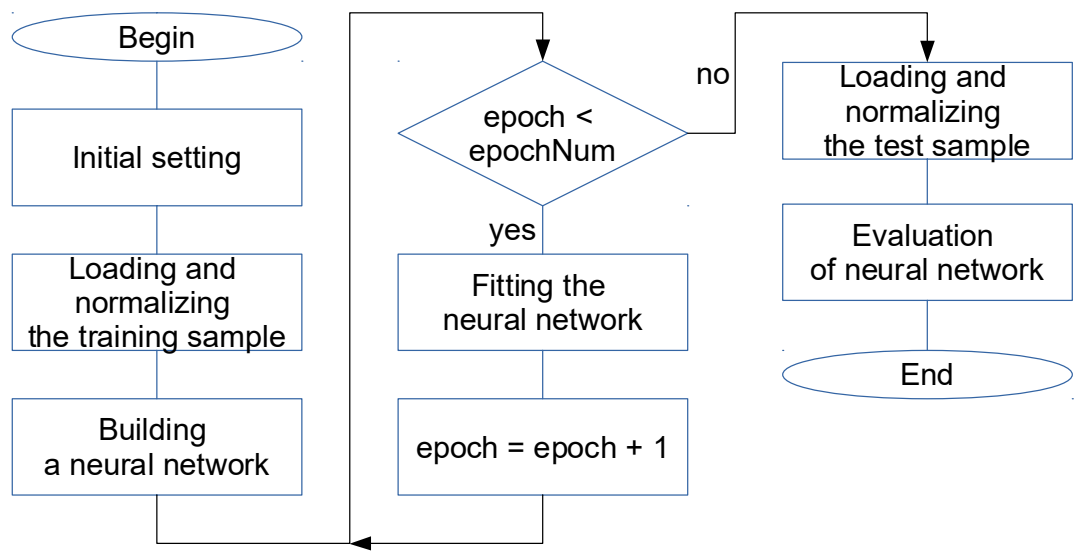

Figure 2. Block diagram used for experiments
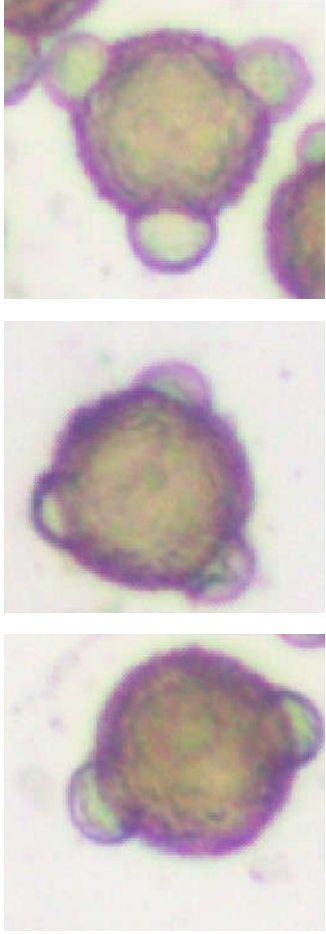

Class 0

(Centaurea scabiosa)
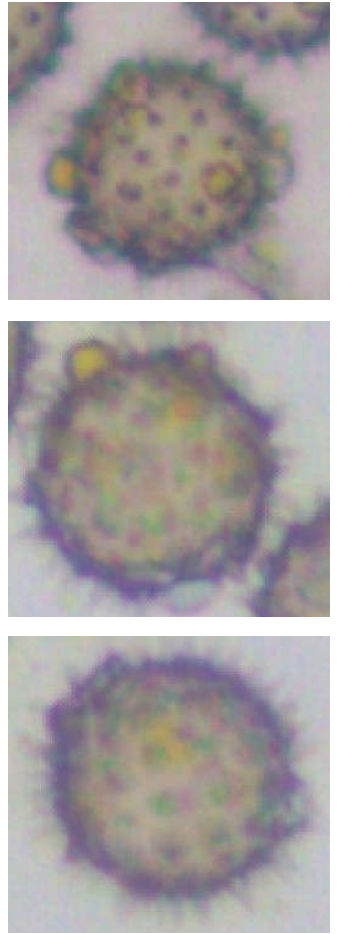

Class 1

(Helianthus)
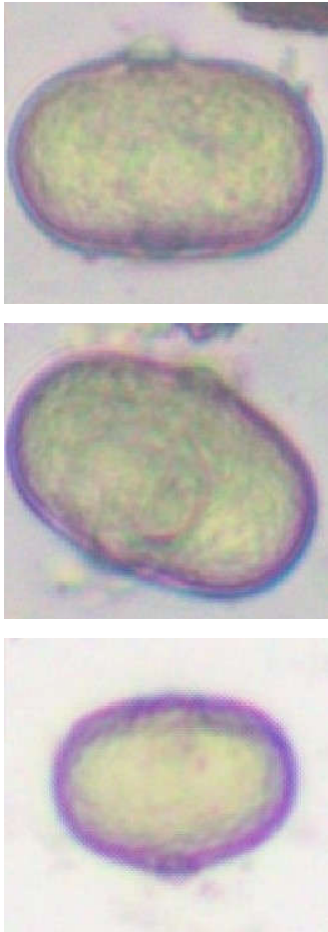

Class 2

(Pisum)
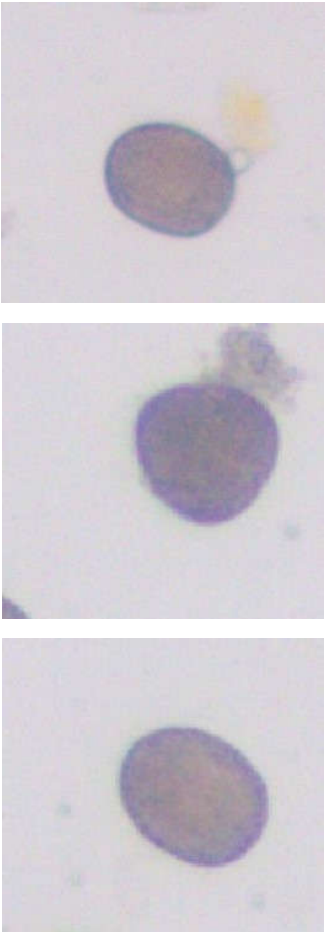

Class 3

(Fagopyrum)

Figure 3. Examples of images used in the experiments

Table 1. Number of images in experiments

\begin{tabular}{c|c|c|c|c}
\hline Sample & Class 0 & Class 1 & Class 2 & Class 3 \\
\hline Training & 352 & 496 & 352 & 320 \\
\hline Test & 88 & 124 & 88 & 80 \\
\hline Total & 440 & 620 & 440 & 400 \\
\hline
\end{tabular}


Korobeynikov A., Kamalova Yu., Palabugin M., Basov I.

"The use of convolutional neural network LeNet for pollen grains classification"

Table 2. Classification results for the test sample

\begin{tabular}{c|c|c|c|c}
\hline \multirow{2}{*}{ Neural network output } & \multicolumn{4}{|c}{ Target output } \\
\cline { 2 - 5 } & Class 0 & Class 1 & Class 2 & Class 3 \\
\hline Class 0 & 79 & 1 & 1 & 1 \\
\hline Class 1 & 1 & 119 & 8 & 0 \\
\hline Class 2 & 8 & 2 & 78 & 2 \\
\hline Class 3 & 0 & 2 & 1 & 77 \\
\hline Total & 88 & 124 & 88 & 80 \\
\hline
\end{tabular}

\section{CONCLUSIONS}

The results obtained in experiments on the test sample showed the applicability and high efficiency of the chosen apparatus of convolutional neural networks for solving the pollen grains classification problem, which is confirmed by high values of classification quality metrics.

In further researches, it is necessary to improve the sample by increasing the number of pollen grain classes, and the number of pollen images in the classes. After that, this neural network can be used in the automatic honey pollen analysis software.

\section{ACKNOWLEDGMENT}

The authors are grateful to the staff "Analytical Center APIS", Ltd. (Moscow region) headed by Elena Yuryevna Balashova for provision of the microscopic images of pollen grains and for active cooperation and consultation.

\section{REFERENCES}

1. Gonçalves, A. B., Souza, J. S., Silva, G. G., Cereda, M. P., Pott, A., Naka, M. H., \& Pistori, H. (2016). Feature extraction and machine learning for the classification of Brazilian savannah pollen grains. PLoS ONE, 11(6), e0157044. doi: 10.1371/journal.pone.0157044.

2. Hwang, G. M., Riley, K. C., Christou, C. T., Jacyna, G. M., Woodard, J. P., Ryan, R. M., ... Masters, D. L. (2013). Automated pollen identification system for forensic geo-historical location applications. In: 2013 IEEE International Conference on Technologies for Homeland Security (HST) (pp. 297-303). Waltham, MA, USA : IEEE. doi: 10.1109/ths.2013.6699018.

3. Stroh, C., Wang, H., Bash, R., Ashcroft, B., Nelson, J., Gruber, H., ... Hinterdorfer, P. (2004). Singlemolecule recognition imaging microscopy. Proceedings of the National Academy of Sciences, 101(34), 12503-12507. doi: 10.1073/pnas.0403538101.

4. Ranzato, M., Taylor, P., House, J., Flagan, R., Lecun, Y., \& Perona, P. (2007). Automatic recognition of biological particles in microscopic images. Pattern Recognition Letters, 28(1), 31-39. doi: 10.1016/j.patrec.2006.06.010.

5. Khanzhina, N., \& Zamyatina, E. (2015). Pollen grains recognition using structural approach and neural networks. Information Models \& Analyses, 4(3), 243-258. Retrieved from http://www.foibg.com/ijima/vol04/ ijima04-03-p03.pdf.

6. France, I., Duller, A. W. G., Duller, G. A. T., \& Lamb, H. F. (2000). A new approach to automated pollen analysis. Quaternary Science Reviews, 19(6), 537-546. doi: 10.1016/s0277-3791(99)00021-9.

7. Marcos, J. V., Nava, R., Cristóbal, G., Redondo, R., Escalante-Ramírez, B., Bueno, G., ... Rodríguez, T. (2015). Automated pollen identification using microscopic imaging and texture analysis. Micron, 68, 36-46. doi: 10.1016/j.micron.2014.09.002.

8. Haas, N. Q. (2011). Automated pollen image classification (Master's thesis, University of Tennessee, USA). Retrieved from https://trace.tennessee.edu/utk_gradthes/1113. 
"Instrumentation Engineering, Electronics and Telecommunications - 2018"

Proceedings of the IV International Forum (Izhevsk, Russia, December, 12-14, 2018)

9. Mander, L., Li, M., Mio, W., Fowlkes, C. C., \& Punyasena, S. W. (2013). Classification of grass pollen through the quantitative analysis of surface ornamentation and texture. Proceedings of the Royal Society B: Biological Sciences, 280(1770), 1-7. doi: 10.1098/rspb.2013.1905.

10. Sinha, N., \& Ramakrishnan, A. (2003). Automation of differential blood count. In: TENCON 2003. Conference on Convergent Technologies for Asia-Pacific Region (pp. 547-551). Bangalore, India: IEEE. doi: 10.1109/tencon.2003.1273221.

11. Daood, A., Ribeiro, E., \& Bush, M. (2016). Pollen recognition using a multi-layer hierarchical classifier. 2016 23rd International Conference on Pattern Recognition (ICPR) (pp. 3091-3096). Cancun, Mexico : IEEE. doi: 10.1109/ICPR.2016.7900109.

12. Kamalova, Y. B. (2014). Calculation of statistical parameters in recognition of pollen grains, obtained by the scanning electron microscope. Intelligent Systems in Manufacturing, 2014(1), 120-123. Retrieved from http://izdat.istu.ru/index.php/ISM/article/download/1540/353 (in Russian).

13. Lomaev, G. V., \& Kamalova, Y. B. (2014). Komp'yuterizirovannyy pyl’tsevoy analiz meda [Computerized pollen analysis of honey]. Pchelovodstvo [Beekeeping], 2014(8), 59-60 (in Russian).

14. Kamalova, Y. B. (2016). Methods and approaches to computerized pollen analysis. Intelligent Systems in Manufacturing, 14(2), 67-70. Retrieved from http://izdat.istu.ru/index.php/ISM/article/download/3275/2083 (in Russian).

15. Goodfellow, I., Bengio, Y., \& Courville, A. (2016). Deep learning. Cambridge, USA : MIT Press. Retrieved from http://www.deeplearningbook.org. 